\title{
ANÁLISE DO DESEMPENHO DO MODELO WRF NA PREVISÃO DE EVENTO SEVERO EM VITÓRIA-ES
}

\author{
Rita C. Feroni*, Elson S. Galvão, Alexandre M. Santiago e Taciana T. A. Albuquerque \\ Departamento de Engenharia Ambiental - UFES \\ *ritaferoni@gmail.com
}

\section{RESUMO}

O trabalho teve por objetivo a análise do desempenho do modelo WRF na reprodução de uma frente de rajada, ocorrida no dia 06 de maio de 2013 em Vitória-ES, causando transtornos e prejuízos à população. Os dados modelados foram comparados com dados medidos nas estações meteorológicas locais, mostrando que o modelo WRF é uma ferramenta eficaz para reprodução de eventos extremos, apesar de subestimar os valores extremos de velocidade do vento.

\begin{abstract}
The study aimed to analyze the performance of the WRF model in reproducing a gust front, which occurred on May 6, 2013, in Vitória-ES, causing inconvenience and loss to the population. The modeled data were compared with data measured at local weather monitoring stations, showing that the WRF model is an effective tool for playback extreme events, despite underestimating the extreme values of wind speed.
\end{abstract}

\section{INTRODUÇÃO}

Segundo Ahrens (2007), uma frente de rajada (gust front) é uma camada de separação entre uma massa de ar fria descendente sob uma massa de ar quente localizada na superfície, que se espalha em todas as direções produzindo rajadas de vento que mudam rapidamente de direção e velocidade.

Dessa forma, esta pesquisa fez uso do modelo Weather Research and Forecast (WRF) (SKAMAROCK et al., 2008) a fim de verificar o fenômeno de frente de rajada que atingiu a cidade de Vitória-ES no dia 6 de maio de 2013, onde os ventos de aproximadamente $76 \mathrm{~km} / \mathrm{h}$ foram registrados pela estação meteorológica do Instituto 
Nacional de Meteorologia (INMET) instalada na Universidade Federal do Espírito Santo (UFES).

\section{METODOLOGIA}

Para as simulações no WRF, foram utilizados quatro grades aninhadas, com o modo two-way ativado, com 43 níveis verticais, onde foram realizados vários testes para identificar o conjunto de parametrizações que melhor representariam o fenômeno estudado (Tabela 1). O período simulado foi de 4 a 7 de maio, $48 \mathrm{~h}$ antes da rajada.

Para validação do modelo, foram utilizados dados medidos por duas estações meteorológicas locais (INMET e Aeroporto) durante o mesmo período simulado.

Tabela 1: Configurações físicas e dinâmicas do modelo WRF

\begin{tabular}{|c|c|c|c|}
\hline Parametrização & $\mathrm{d} 01(27 \mathrm{~km}) \quad \mathrm{d} 02(9 \mathrm{~km})$ & $\mathrm{d} 03(3 \mathrm{~km})$ & $\mathrm{d} 04(1 \mathrm{~km})$ \\
\hline Dimensões & $100 \times 100$ & $100 \times 100$ & $64 \times 82$ \\
\hline Microfísica & Thompson & \multicolumn{2}{|c|}{ WRF Single-Moment 6-class } \\
\hline Radiação OL & \multicolumn{3}{|c|}{ Rapid Radiative Transfer Model } \\
\hline Radiação OC & \multicolumn{3}{|c|}{ Dudhia scheme } \\
\hline CS & \multicolumn{3}{|c|}{ MM5 similarity } \\
\hline CLP & \multicolumn{3}{|c|}{ Pleim-Xiu surface layer } \\
\hline Cúmulus & Grell 3D & B.M.Janjic & nenhum \\
\hline Camada de solo & \multicolumn{3}{|c|}{4} \\
\hline Dados Sinóticos & \multicolumn{3}{|c|}{$\mathrm{NCEP} / \mathrm{NOAA}-1^{\circ} \times 1^{\circ}$} \\
\hline Dados Relevo & \multicolumn{3}{|c|}{ USGS - Gtopo30 } \\
\hline
\end{tabular}

\section{RESULTADOS E DISCUSSÃO}

A partir das Figuras 1.a e 1.b, pode-se observar que em geral o modelo conseguiu representar o comportamento da velocidade do vento, porém mostrou-se limitado para velocidades superiores a $9 \mathrm{~m} / \mathrm{s}$ no momento em que a rajada atingiu valores de velocidade acima deste, com pico de aproximadamente $21 \mathrm{~m} / \mathrm{s}$ e $14 \mathrm{~m} / \mathrm{s}$ para as estações do INMET e do aeroporto, respectivamente. 
As Figuras 1.c e 1.d mostram os resultados para a direção predominante do vento, podendo observar que apesar do modelo não reproduzir satisfatoriamente a intensidade do vento durante o evento ele foi capaz de representar com bom desempenho a inversão na direção do vento de norte-noroeste $(\mathrm{N}-\mathrm{NW})$ para sul-sudoeste $(\mathrm{S}-\mathrm{SW})$. Através da Figura 2, é possível notar a queda brusca na temperatura de aproximadamente $10{ }^{\circ} \mathrm{C}$ em menos de uma hora.
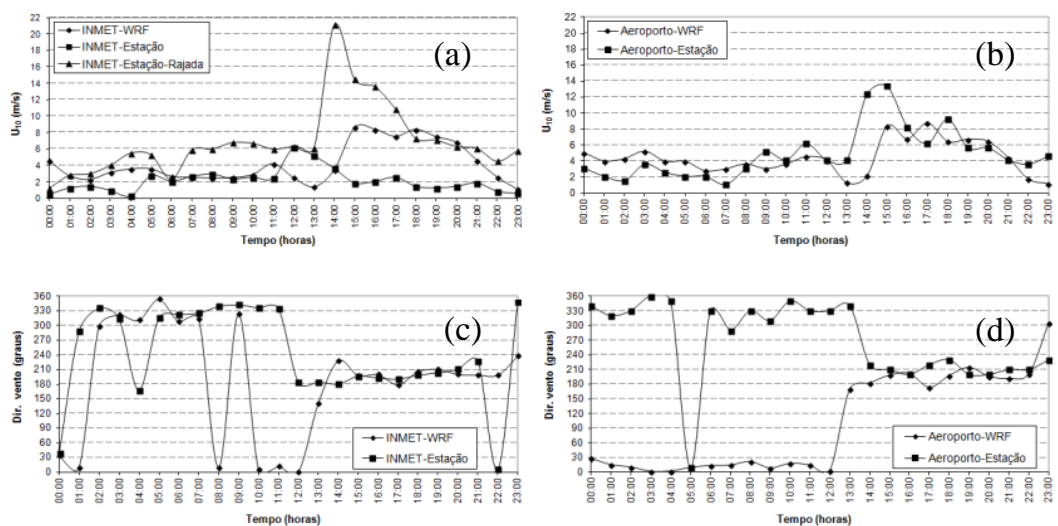

Figura 1. (a) Vel. vento (WRF) e estação INMET; (b) Vel. vento (WRF) e Aeroporto; (c) Dir. vento (WRF) e estação INMET; (d) Dir. vento (WRF) e estação INMET.

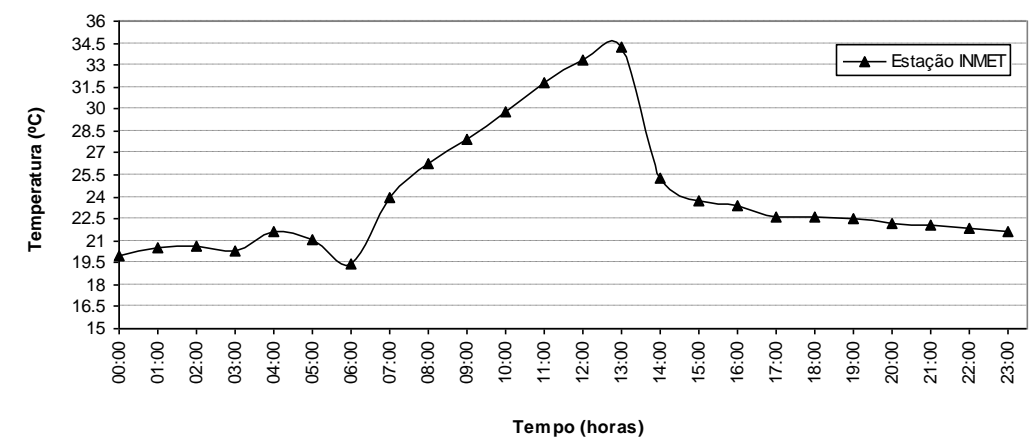

Figura 2. Perfil de temperatura no dia 06/05/2013 na estação INMET.

As Figuras 3.a e 3.b mostram as rosas dos ventos para o dia de estudo nas estações INMET e aeroporto. A Figura 3.c mostra a rosa dos ventos para maio (série de dez anos), com vento predominante S-SW. A mudança de direção observada nas Figuras 1.c e 1.d de NNW para direção S-SW a partir do período das 13:00 horas, associada aos dados de temperatura da Figura 2 e de direção do vento nas Figuras 3, indica a entrada de uma frente fria na região. Tal afirmação é sustentada pelo comportamento da direção do vento em relação ao aumento dos ventos, com rajada de aproximadamente $76 \mathrm{~km} / \mathrm{h}(21 \mathrm{~m} / \mathrm{s})$, velocidade característica de frentes frias (STECH e LORENZETTI 1992). 


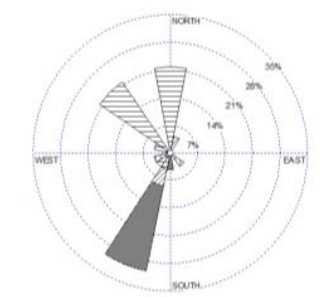

Velocidade do vento $(\mathrm{m} / \mathrm{s})$

Шा $0,5-2,1$

(a)

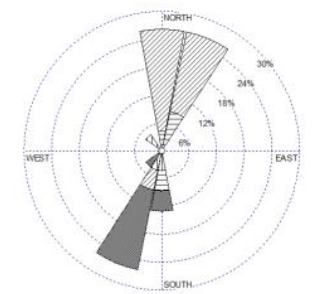

$5,7-8,8$

(b)

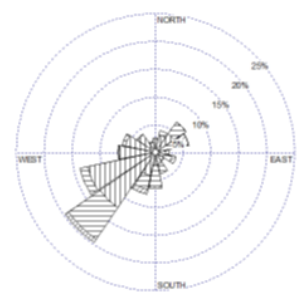

$8,8-11,1 \square \gg=11,1$

(c)

Figura 3. Rosa dos ventos para o dia 06/05/2013 com dados modelados para a estação INMET (a) e Aeroporto (b); e série histórica da região (10 anos).

\section{CONCLUSÕES}

Este trabalho apresentou uma validação do modelo WRF em um evento ocorrido em maio de 2013 em Vitória-ES. O modelo conseguiu prever o perfil de variação da velocidade do vento, porém não capturou os valores máximos de velocidade. O WRF mostrou-se uma ferramenta eficaz em relação às mudanças na direção predominante do vento, capturando a chegada da frente fria, com mudanças bruscas na direção e intensidade da velocidade do vento no horário em que ocorreu a rajada de vento seguida de uma frente fria.

\section{AGRADECIMENTOS}

Á CAPES e CNPQ, pelo apoio financeiro.

\section{REFERÊNCIA BIBLIOGRAFICA}

\section{AHRENS, C.D. Meteorology today - an introduction to weather, climate and} environment. $8^{\mathrm{a}}$ ed. Thomson Brooks/Cole. 2007.

SKAMAROCK, W. C. et al.. Description of the Advanced Research WRF Version 3.

National Center for Atmospheric Research Boulder, Colorado, USA, 2008.

STECH, J. L; LORENZETTI, J. A. The response of the South Brazil Bight to the passage of intertime cold fronts. Journal Geophysical Research, v. 97, n. 66, p. 9507-9520, 1992. 